\title{
Presentimento di una religione senza dio in Friedrich Feuerbach*
}

\author{
Pressentimento de uma religião sem deus \\ em Friedrich Feuerbach
}

Pressentiment of a godless religion

in Friedrich Feuerbach

DOI: https://doi.org/10.20873/rpv6n1-81

Ferruccio Adolfi

Email: ferruccio.andolfi@unipr.it

\begin{abstract}
Sommario
Il saggio lavora l'ipotesi che negli scritti che Friedrich Feuerbach dedica alla religione del futuro e alla chiesa del futuro possa trovarsi qualche elemento di riflessione utile ad articolare il tema, oggi assai discusso, di una religione senza dio. Per un verso questo esercizio sottolinea la prospettiva umanistica della filosofia in questione, per l'altro verso destaca il bisogno di creare una nuova forma di legame capace di diffondere una fede che unisca gli uomini con un vincolo più forte di quello del cristianesimo, cioè che promuova e sostenga l'aspirazione, che ai nostri giorni si manifesta in molteplici modi, a integrare la insufficienze delle singole forze con uno spirito comune. Infine, si tratta sempre dell'amore del prossimo fondato sulla nostra "natura comune", particolarmente sul reciproco bisogno che proviamo l'uno dell'altro e che ci spinge a considerarci "fratelli e sorelle nell'uomo".**
\end{abstract}

\section{Parole-chiave}

Friedrich Feuerbach. Religione del futuro. Religione senza dio. Atei devoti. Umanesimo.

\section{Resumo}

* Conferência ministrada em Münster, Alemanha, em 11 de novembro de 2016.

${ }^{* *}$ Resumo elaborado pela equipe editorial. 
0 artigo trata da hipótese de que nos escritos de Friedrich Feuerbach sobre a religião do futuro e sobre a igreja do futuro possamos encontrar elementos de reflexão úteis para a articulação do tema, hoje tão discutido, de uma religião sem deus. De um lado, esse exercício sublinha a perspectiva humanista da filosofia em questão e, por outro, destaca a necessidade da criação de uma nova forma de laço capaz de difundir uma fé que una os homens com um vínculo mais forte que o vínculo do cristianismo, isto é, que promova e sustente a aspiração, que nos nossos dias se manifesta de modos diversos, a integrar as insuficiências das forças individuais com um espírito comum. Trata-se sempre do amor do próximo fundado sobre a nossa "natureza comum", particularmente sobre a recíproca necessidade que experimentamos uns dos outros e que nos leva a considerar-nos "irmãos e irmãs no homem".

\title{
Palavras-chave
}

Friedrich Feuerbach. Religião do futuro. Religião sem deus. Ateus devotos. Humanismo.

\begin{abstract}
The article deals with the hypothesis that in Friedrich Feuerbach's writings on the religion of the future and on the church of the future, we can find elements of reflection useful for articulating the theme, so much discussed nowadays, of a religion without god. On the one hand, this exercise underlines the humanist perspective of the current philosophy and, on the other hand, it highlights the need to create a new form of union capable of spreading a faith that unites men with a stronger bond than Christianity, that is, which promotes and sustains the aspiration, which today manifests itself in different ways, to integrate the insufficiencies of individual forces with a common spirit. It is always about the love of the neighbor founded on our "common nature", particularly on the reciprocal need that we have experienced from each other and that leads us to consider ourselves "brothers and sisters in man".
\end{abstract}

\section{Keywords}

Friedrich Feuerbach. Religion of the future. Religion without god. Devout atheists. Humanism.

1. Questo saggio nasce dall'ipotesi che negli scritti che Friedrich Feuerbach ha dedicato alla religione del futuro e alla chiesa del futuro possa trovarsi qualche elemento di riflessione utile ad articolare il tema, oggi assai discusso, di una religione senza dio. ${ }^{1}$

\footnotetext{
${ }^{1}$ Tre scritti di Friedrich Feuerbach hanno lo stesso titolo, Die Religion der Zukunft. Il primo quaderno fu pubblicato a Zürich nel 1845 dal Verlag des literarischen Comptoirs, gli altri due a Nürnberg nel 1845 dal Verlag von Theodor Cramer, rispettivamente con il sottotitolo Die Bestimmung des Menschen e Mensch oder Christ? Verranno citati con la sigla RdZ, seguita da un numero romano (I, II e III), e poi da un numero arabo che indica il paragrafo. Per Die Kirche der Zukunft, Bern 1847, utilizzo la sigla KdZ.
} 
La questione di una separazione del sacro dalla fede in un dio trascendente ha dietro di sé una lunga storia, nella quale l'evoluzione della teologia si intreccia con la sua critica. Così nella teologia liberale di Schleiermacher il sentimento dell'infinito, in ogni sua forma, viene assunto come il nucleo vero e proprio della religione, mentrel'ammissione dell'esistenza di dio non viene considerata altrettanto essenziale (Reden, 1799, secondo discorso). Poco più tardi Ludwig Feuerbach indica nell'essenza umana il segreto e insieme la veritàdella religione. Il rapporto etico dell'uomo con l'uomo ha per lui le credenziali per soppiantare la religione. E tuttavia, stimolato dalle critiche di Stirner, egli accetta di essere annoverato tra gli "atei devoti", che conservano i predicati di dio, anche se negano la suasoggettività indipendente. Essi si attengono alla sola religione che rimarrà, quella per cui "aver religione vuol dire pensare agli altri" e non averla pensare solo a se stessi (L. FEUERBACH, 1970, p. 436). Non rigetta l'obbiezione di Stirnerdi aver ridotto la religione ad etica (o l'etica a religione), ma precisa che si tratta di un'etica, che, diversamente da quella kantiana e del protestantesimo illuminato, assume come misura l'uomo. Sacri, spiega, non sono i rapporti morali come tali, bensì l'uomo che ne è il termine. Il fenomeno più che morale del perdono è addotto come simbolo di una morale umanistica che eredita dal cristianesimo un atteggiamento conciliativo anziché giudicante (L. FEUERBACH, 1970, p. 440). Quando, qualche decennio più tardi, Nietzsche proclama la "morte di dio" e annuncia il superuomo, simuove anche lui nell'orizzonte di una trasfigurazione del sacro piuttosto che in quello della totale immanentizzazione tentata vanamente da Stirner.

Sulle linee tracciate da questi precursori si muovono quei teologi e critici della religione contemporanei che riflettono in diverse chiavi sulla possibilità di una "religione senza dio", un'espressione che nel tempo ha perso quel sentore provocatorio che poteva aver avuto originariamente. Di recente il teologo madrileno Manuel Fraijo nel suoReligion sin Dios? ha ricostruito sapientemente il percorso che nella modernità ha condotto a immaginare una “religione senza Dio”. Sebbene egli approdi infine a una riabilitazione di Dio con argomenti che possiamo considerare una variante di quelli kantiani - per restituire un qualche senso alle 
esistenze fallite e ingiustamente dimenticate è necessario postulare un dio che assicuri qualche forma di risarcimento - il suo sguardo nei confronti dell'“ateismo devoto" è di piena comprensione. ${ }^{2}$ Lo scritto di Fraijo ci richiama a quella dimensione della "salvezza" che nella religione ha avuto sempre un ruolo essenziale. È probabile che in questo egli si consideri erede di unaltro grande pensatore spagnolo, Unamuno, che in Del sentimientotragico de la vida (1913) considera la fede nell'immortalità personale un elemento centrale dell'animo religioso. È in nome di un diritto irrinunciabile all'affermazione di sé che la coscienza credente si ribella all'evidenza del proprio destino mortale.

A un secolo di distanza tuttavia questa fede ha perso plausibilità. Lo spirito religioso sopravvive in altre forme, confrontandosi soprattutto col naturalismo scientifico e con le spiegazioni neuroscientifiche dei sentimenti morali. Nello scritto postumo di Dworkin, Religion without God (2013), la fede nell'oggettività dei valori è trattato come un equivalente umanistico-religioso della fede in un dio trascendente, capace anche di garantire un'alleanza tra credenti e non credenti. La curvatura etica di tale "religione senza dio" è indubbia, anche se nel rapporto con l'universo essa riacquista il respiro e la profondità del "numinoso". In Roger Scruton (2013), il rapporto interpersonale io-tu è di nuovo centrale, e "la presenza reale di Dio diventa un caso speciale della presenza reale del soggetto umano", tuttavia alcune categorie tipiche del pensiero religioso, e più in particolare del cristianesimo, vengono conservate. L'agape è "lo spirito che dice sempre di sì, percorrendo il sentiero del dono e del sacrificio" e quest'accettazione della sofferenza e del sacrificio può essere giustamente descritta come “redenzione”. L'essere appare come un dono alla persona religiosa, che sente il bisogno di ringraziare. Questo bisogno di rendere grazie è condiviso da una comunità, almeno ideale, e si rende manifesto in momenti di passaggio, attraverso apposite cerimonie (cf. SCRUTON, 2013, p. 164-167). Queste posizioni hanno accenti diversi, ma differiscono in modo sostanziale da quelle di pensatori di matrice radicalmente illuminista, quali Michel Ofray, Richard Dawkins o

\footnotetext{
${ }^{2}$ Isegoria, 47, 2012/2, p. 381-419. Il saggio è stato tradotto in italiano nel n. 50 (2014/2) de La società degli individui, dove è seguito dal commento di numerosi studiosi.
} 
Paolo Flores D'Arcais, che proclamano senza alcuna titubanza l'irrazionalità della credenza in dio e magari predicano una "scristianizzazione radicale della società" come condizione per attribuire valore alla vita terrena. ${ }^{3}$

2. Il primo dei tre scritti di Friedrich Feuerbach intitolati Die Religion der Zukunft (1843), contiene, la cosa è abbastanza singolare,lo sviluppo di un'etica eudemonistica a cui il fratello Ludwig sarebbe arrivato in forma aperta due decenni più tardi, in Spiritualismus und Materialismus (1866) e negli appunti inediti Zur Moralphilosophie. In questo caso si direbbe che Friedrich faccia da battistrada. Nelle opere di Ludwig l'istinto di felicità era stato confinato dapprima all'ambito della religione. Ancora nell'Essenza del cristianesimo esso non sembra libero da questa ipoteca religiosa. Alla filosofia viene rivendicata la funzione teoretica di contemplare la realtà per se stessa e non in vista di interessi pratici, riconducibili appunto al desiderio di felicità.

Ora nello scritto che stiamo esaminando Friedrich riconosce che l'istinto di felicità costituisce l'anima inconscia del cristianesimo (I, 32), ma questa osservazione non ha l'obiettivo di trascendere quest'impulso, bensì di riconoscere la sua piena giustificazione. Se nonviene "sviato" esso è "il cuore dell'umanità stessa" (I, 32). È attorno a questo nucleo di valori etici che viene abbozzata, nella prefazione, la stessa definizione di religione del futuro: "conoscenza della vera condizione fondamentale della natura umana, divenuto disposizione interiore che penetra e determina l'uomo intero".

Lo sviamento dell'istinto di felicità verso una meta immaginaria (I, 3) si dà quando la salvezza è fatta dipendere da un dio personale. Nell'infanzia Friedrich ricorda come viceversa proprio l'elemento personale gli fosse apparso autentico nella vita religiosa. Nei Ricordi della mia vita religiosa, pubblicati in appendice, egli appare portatore di un bisogno religioso che si appaga nel rapporto con un dio padre fondamentalmente clemente. La crisi religiosa viene

\footnotetext{
${ }^{3}$ Cf. DAWKINS, 2006; ONFRAY, 2006; FLORES D'ARCAIS; ONFRAY; VATTIMO, 2007.
} 
messa in moto, alla vigilia della Cresima, dalla difficoltà di tenere insieme questaimmagine con quella dell'insegnamento catechistico di un dio trino, in cui la conciliazione di dio con una natura umana corrotta è resa possibile dal sacrificio di Cristo, cioè di una persona diversa dal padre. Questi ora appare spietato nel chiedere una riparazione di sangue dal suo stesso figlio e distrugge l'immagine del dio compassionevole e amato della sua fanciullezza. ${ }^{4}$ Altrove Friedrich spiega come questa evoluzione dalla raffigurazione di un dio "amabile" a uno "terribile" sia inevitabile per l'animo religioso, che pur di assicurarsi l'eterna beatitudine preferisce attenersi al caso peggiore di un dio pericoloso da rabbonire con ogni genere di sacrifici (KdZ, 48-49).

Lo scritto contiene i lineamenti fondamentali di un'antropologia che intende poggiarsi, in modo equilibrato, sul "cuore". La natura umana è descritta come un insieme di facoltà: lo spirito autocosciente, la volontà e la sensibilità, che trovano un'armonizzazione grazie al dominio della ragione (I, 12-13). Questo dominio peraltro consiste solo nella sua prospettiva (Aussicht) che assicura un' attività armonica delle forze umane. Non si tratta di assegnarle un ruolo privilegiato bensì di riconoscere la funzione regolatrice che solo essa è in grado di esercitare (I, 14).

Lo sviamento dell'istinto di felicità verso mete fantastiche comporta una rinuncia alla lotta di liberazione della vita dai mali reali, quegli effetti di "alienazione" che le analisi del suo più noto fratello ci hanno reso familiari. Tra questi effetti Friedrich segnala il "turbamento" dell'animo religioso, che compromette la "pace dell'anima" (I, 20-21). Solo il rapporto verso l'umanità e la determinazione a rendersi utile per essa è in grado di procurare al pensatore quella paceinteriore che è preclusa all'uomo pio.

Resta piuttosto incerto il motivo per cui una simile visione del mondo, etico.antropologica, debba essere definita religiosa. Il fatto che sia una modificazione della ricerca di felicità che è stata all'origine delle formazioni religiose del passato è sufficiente a

\footnotetext{
${ }^{4}$ Sulla scellerata assurdità che ha reso doveroso per gli uomini offrire a dio in olocausto i propri figli cfr. anche RdZ, II, 14.
} 
giustificare questapretesa? Qui, come altrove, Friedrich Feuerbach si rifiuta di assumere verso le religioni del passato, e specialmente verso il cristianesimo, quell'atteggiamento denigratorio che è divenuto piuttosto diffuso nella cultura laica di matrice illuministica dei nostri giorni. Il fatto che il cristianesimo sia un'aberrazione (Verirrung) dell'istinto di felicità insito nel cuore dell'uomo non comporta che esso debba essere dissolto violentemente, perseguitato e rimesso al disprezzo del popolo(I, 32). L'umanità colta dovrebbe porsi nei suoi confronti "nel medesimo rapporto di un uomo maturo rispetto a sua madre, che egli onora come propria madre, sebbene si ricordi di alcune ingiustizie ed errori a cui fu indotta per ignoranza e semplicità nell'educazione dei figli" (I, 32). Anche questo atteggiamento comprensivo della tradizione non giustifica però di per sé una caratterizzazione religiosa della nuova visione del mondo.

3. Nel secondo scritto (Die Religion der Zukunft, 1845) troviamo qualche chiarimento circa la relazione tra la vita etica e la religione annunciata. La categoria di "sacro" è continuamente richiamata, ora però in rapporto alla natura umana e a tutto quanto le appartiene. A cominciare dall'istinto di felicità che è il suo modo di affermazione. "Giustificato e perciò sacro è in tutti gli uomini il desiderio di essere felici" (II,8), che è un bisogno universale ed eterno (II, 13). Se sacro è il desiderio, sacra è anche la sua meta: la salvezza dell'umanità (II, 9).

Su questo desiderio si innesta l'intera vita morale, che ha quindi, dobbiamo intendere, una base eudemonistica. Friedrich non si confronta, come avrebbe fatto più tardi Ludwig, con l'etica del dovere kantiana e con la morale della compassione schopenhaueriana, ma si limita ad appellarsi a una specie di consensus gentium, o almeno dei popoli "civilizzati" circa le norme di comportamento morale: rispettare la vita come "sacra e inviolabile", non ricambiare il male, soccorrere gli altri, conoscere e governare le proprie inclinazioni (II, 16).

In ultimo le leggi morali si fondano sulla reciproca indigenza (Bedürftigkeit) degli uomini, che per raggiungere i loro obiettivi essenziali hanno bisogno gli uni degli altri. Ma 
questo stato di bisogno reciproco fornisce anche la base del primo principio della religione del futuro, che proclama sacro e giustificato l'istinto di felicità di tutti gli uomini (II, 15 e 13).

Il paragrafo 23 fa il punto sulla relazione tra religione ed etica. Essesi riferiscono a un medesimo oggetto (l'uomo) e nella religione del futuro si raggiunge una relazione particolarmente stretta di fede e moralità. "La religione - si legge - è la fede nella giustificazione e santità del desiderio umano di essere felici, nella santità delle condizioni per il soddisfacimento di questo desiderio fondate nella natura umana". La vita etica è invece (non è che) "la conferma di questa fede nelle relazioni sociali degli uomini". Secondo un altro approccio, la religione del futuro riconosce la volontà dell'essere umano nella sua intrinseca santità, mentre "la vita etica mira alla realizzazione di questa volontà nella società". 0 , con una metafora, "la vita etica è il fiore della religione del futuro", e il frutto di questo fiore è il bene della società (II, 23). Il tratto differenziale tra l'una e l'altra sembrerebbe dunque che la prima (la religione) consiste in una credenza e intuizione del singolo, e l'altra invece (la vita etica) traducein atto, nella vita sociale, quella credenza.

Nella concezione etica tratteggiata in queste pagine il bene dell'individuo e quello comune si fondono in perfetta reciprocità. Ciascuno dei due termini - individuo e società non deve ricevere "troppo né troppo poco". Il giusto tatto per l'aspirazione alla felicità personale farà sì che essa si realizzi senza danno per gli altri (II, 27). (Analogamente Ludwig sosterrà che accanto a un istinto di felicità diretto al bene proprio esiste in ciascuno di noi un istinto di felicità indirizzato al bene comune.)

Nella vera religiosità e moralità il sentimento del proprio valore va sempre congiunto a quello della propria dipendenza dagli altri (II, 29). Per la vita etica si richiede la rappresentazione del bisogno reciproco, che mi fa riconoscere nell'altro uomo un dio: quel sentimento che il cristiano reprime perché come tale non ha bisogno degli uomini (II, 30-31). La filantropia gli è estranea perché il credente non ama il prossimo per amore dell'uomo ma solo per amore di dio, "in vista della salvezza personale della propria anima" (II, 36). L'opposizione del cristianesimo ai principi della religione del futuro non potrebbe essere più 
radicale. Per esso la natura umana è condannata anziché essere rispettata come sacra. E non è in gioco la salvezza dell'umanità bensì quella del singolo uomo, non la salvezza terrena ma quella nell'aldilà, e della sola anima (II, 39). Il disconoscimento del bisogno reciproco degli uomini, che costituisce la base della moralità, porta con sé una svalutazione in generale delle leggi morali, che difatti nel cielo cristiano vengono "dissolte" (aufgelöst).

La preoccupazione di salvaguardare un universo normato da regole prevedibili, e non dall'arbitrio della volontà divina, impedisce a Friedrich di apprezzare un lato per così dire "anarchico" del cristianesimo, che garantisce l'indipendenza del credente da sistemi dinorme razionali e in generale da ogni autorità. Se Dio è la proiezione di se stessi, o del proprio io migliore, appellarsi unicamente a lui comporta anche la resistenza a qualsiasi altra regola o l'impegno costruttivo a creare regole non uniformizzanti. Nell'universo razionale-morale di Friedrich invece le istituzioni, a partire dallo Stato, avanzano la giusta pretesa di essere sostenuti. "L'autorità sarà giustificata ad accrescere le sue pretese sul popolo e ad esigere da essodi essere sostenuta al meglio nelle sue imprese utili con fiducia ecollaborazione" (II, 45). La religione del futuro non presenta attrattive per gli "animi pigri" che preferiscono abbandonarsi alla provvidenza, ma neppure per gli "animi fieri" che trovano opprimente il sentimentodi dipendenza dai loro simili" (II, 44). Un governo potrà contare di più su un popolo compenetrato di rispetto e gratitudine che su un popolo ingrato (II, 45).

Che ne sarà in futuro del rapporto sentimentale che i singoli uomini intrattengono con l'essere sovrumano, autocosciente e personale posto a fondamento di ogni essere? Dove esso è diventato abitudine e bisogno, a causa dell'educazione cristiana, combatterlo sarebbe uno sforzo vano, risponde Friedrich, tollerante. Purché, aggiunge, non diventi un punto centrale nella vita attiva e pretenda di valere come motivazione morale, riducendo la storia a un "teatro di marionette". Ilrapporto sentimentale sopravvive in modo orizzontale nei legami sociali, che sono legami di fiducia, rispetto e gratitudine. Ma si fonderà in avvenire su "conoscenze assolutamente chiare e distinte" (II, 47). 
Questa medesima esigenza di chiarezza e distinzione porta Friedrich a mettere in forse l'altro grande pilastro delle religioni del passato, la fede nell'immortalità, che è, al pari della divinità, "unalanda sconosciuta e oscura" (I, 48). Da questa credenza è inutile attendersi, come si fa abitualmente, una "consolazione", visto che rappresenta piuttosto un'evasione dal compito di affrontare, con mezzi adeguati, le difficoltà della vita. Solo l'ascolto da parte di altri e ilpassare del tempo sono rimedi appropriati ai dolori dell'anima. "Quando un dolore comincia ad ascoltare una consolazione è già sulla via della guarigione" (I, 48).

4. La fede nell'immortalità nella storia delle idee religiose, almeno nel mondo occidentale, è sempre andata di pari passo con l'altra fondamentale credenza nell'esistenza di Dio. Secondo l'analisi di Ludwig Feuerbach costituirebbe anzi il senso ultimo e vero della fede in Dio, che dell'immortalità dell'anima e della sua eterna felicità dovrebbe essere garante (...). Nella Critica della ragion pratica di Kant la reciprocità delle due credenze diventa particolarmente evidente. L'adempimento sempre parziale degli obblighi morali, e la non corrispondenza della felicità alla virtù, portano a postulare un'esistenza dell'anima al di là della morte in cui progressivamente la realizzazione perfetta del bene - e del "sommo bene", che esige l'adeguamento di virtù e felicità - possa compiersi, e di un essere onnipotente che consenta questa pienezza.

Ma l'immortalità della persona è stata vista come quintessenza della religione non solo in questa prospettiva razionalistica, bensì anche da una vitalistica, che parte, come nel caso ricordato di Unamuno, dal rilievo della radicale inquietudine degli esseri umani e della loro ribellione rispetto alla mortalità avuta in sorte. "Ciò a cui anelo non è naufragare nel grande Tutto, nella Materia o nella Forza infinite ed eterne, o in Dio; ma possederlo, divenire io stesso Dio senzacessare di essere quell'io che ora afferma tali cose" (UNAMUNO, 2017, p. 84).

L'ancoraggio ad una ipotetica vita ultraterrena comunque fondata non è tuttavia l'unico modello possibile di religiosità. Già dal primo Ottocento, con Schleiermacher, è stata introdotta l'ipotesi che la categoria del religioso possa emanciparsi dalla credenza nell'esistenzadi dio e 
della salvezza dell'anima, che anzi, per le sue implicazioni "utilitaristiche", potrebbe essere meno autentica di quel sentimento dell'Assoluto o dell'infinito a cui la religione dovrebbe essere più propriamente riportata (Discorsi sulla religione). L'elemento personale delle religioni viene trasceso. La "salvezza", ammesso che sia ancora possibile utilizzare questo concetto, viene cercata in un rapporto di unione quasi mistica con la totalità del reale, che assicura all'io una paradossale eternizzazione. In questa tradizione un punto di riferimento costante è il Deus sive natura di Spinoza.

Ci si può chiedere quale fisionomia assuma in Friedrich Feuerbach la sopravvivenza del sacro oltre l'orizzonte non più difendibile del Dio personale onnipotente. Si può dire che anch'essa si iscriva all'interno del vasto fenomeno delle religioni senza dio? Abbiamo visto come i confini tra religione e vita etica diventino in lui estremamente sfumati. Ora, invece, se dobbiamo accogliere un suggerimento di William James in The Varieties of Religious Experience (1902), il tratto tipico dell'animo religioso è proprio quello di tenere ferma la distinzione.

5. Il terzo saggio sulla religione del futuro (1845) è noto anche con il titolo Mensch oder Christ? ovvero Sein oder Nichtsein? L'opposizione tra le due visioni del mondo non potrebbe essere più netta, ha a che fare con il livello primario dell'esistenza, con l'impulso vitale, affermato o negato, che è istinto di felicità. Una grande lotta si profila tra l'universo della fede in un essere trascendente che implica il disconoscimento della natura umana ed è la radice di ogni miseria terrena e la visione di un mondo di uomini che riconoscono reciprocamente il diritto alla felicità di ogni altro (III, 6) e cooperano albenessere comune mediante il lavoro un lavoro gradevole eattraente - e un'attiva filantropia (III, 12). Per il miglioramento della condizione umana uno spirito diverso da quello del cristianesimo devedominare l'umanità (III, 6). L'umanesimo, cioè l'annuncio (Botschaft) che l'essenza umana trova in se stessa il proprio baricentro subentraal posto dei sistemi religiosi dominanti (III, 11).

La tensione spirituale sovrumana generata dalla rappresentazione di un essere spirituale superiore alla natura umana porta alla negazione (repressione) della sensibilità, 
mentre la rappresentazione di una condizione beata nell'aldilà elimina il gusto per la vita e perogni attività riferita al miglioramento dei nostri rapporti terreni.

Queste osservazioni si collocano nel contesto di un progetto di riforma culturale ed educativa che ha tonalità missionarie. È urgente ilcompito di "escludere totalmente la dottrina di dio dall'educazione dell'umanità" (III, 17). Anche l'educazione deve emanciparsi, comeogni altra scienza, dalla teologia e dalla chiesa (III, 18).

L'idea di chiesa viene però mantenuta. A conclusione del saggio Friedrich Feuerbach dà qualche indicazione su come debba essere reinterpretato questo concetto. Al posto dell'istituzione finora così denominata subentrerà un sistema di Unioni (Vereine) che sorgeranno in vista di determinati scopi utili. Incidentalmente ricordo che il termine Verein compare nell'Unico di Stirner per designare asso - ciazioni volontarie prive dell'aura sacrale che circonda la comunità (Gemeinschaft). Ma per Friedrich invece queste due figure non ci pongono in altenativa: la unioni rimandano a "una unione più comprensiva" che ha la finalità di "promuovere, con la parola e l'azione, in uno spazio pubblico, tutto ciò che è necessario per la soddisfazione e la formazione dell'uomo intero e per l'allevamento diuna specie più felice". Ciò che egli designa "chiesa del futuro" è appunto una tale unione, che fornisce un asilo all'umanità sofferente. Essa stimola "la coscienza della nostra reciproca indigenza, la coscienza della nostra forza nell'azione comune per scopi di utilità sociale, lo spirito della giustizia e della fiducia, il senso del bene comune" e la "realizzazione" stessa di questo bene (III, 19).

6. Nella prospettiva umanistica dei fratelli Fuerbach nonsembrerebbe esserci spazio per nessuna Chiesa. Come dobbiamo interpretare allora le riflessioni di Friedrich in Die Kirche der Zukunft? Lo scritto è in gran parte dedicato in effetti alla demolizione del principio teologico e delle istituzioni che lo sorreggono. Una riforma interna alla Chiesa, o alle Chiese cristiane, nella direzione della "mondanizzazione" auspicata (KdZ, 52) non sembrava d'altronde prevedibile, benché in seguito si sia effettivamente compiuta, sia pure con estrema prudenza. 
La nuova Bibbia è pensata come una summadei risultati culturali più alti che l'umanità ha raggiunto, non certo come un nuovo testo sacro.

Resta il fatto che l'evoluzione dell'umanità è presentata come un seguito di svolte religiose, che toccano un livello primario dell'esistenza. La stessa resistenza che le religioni tradizionali offrono al cambiamento induce a pensare che questo non possa compiersi, oalmeno compiersi con sufficiente radicalità, se non in nome di una credenza forte, la fede nell'uomo e nella sua salvezza, che eredita trattidelle religioni contestate. Quando Gustav Radbruch nei Feuerbachsscrive che Friedrich "predicava ciò che suo fratello Ludwig insegnava"(predigte, was sein Bruder Ludwig lehrte), è probabile che abbia di mira questa circostanza (RADBRUCH, 1942, p. 175).

La visione del mondo proposta presenta tratti latamenteevoluzionistici. Si suppone che la natura possa funzionare ed essere spiegata iuxta propria principia ( $\mathrm{KdZ} 16)$. Essa va concepita come un tutto organico che esiste per se stesso eternamente e necessariamente(KdZ 30). La fragilità e contingenza degli eventi mondani non puòessere soddisfacente per gli uomini, che cercano un "punto di sosta" e privilegiano ciò che permane rispetto a ciò che è transeunte (KdZ 20-21). Ma non c'è ragione per appoggiarsi a un essere eterno fuori del mondo. Certi elementi e forze della natura sono eterni e dal loro gioco è possibile derivare la varietà dei fenomeni. Friedrich non vede difficoltà neppure nel derivare lo spirituale dal sensibile, mentre non trova nessuna attestazione di un processo contrario, che faccia sorgereil sensibile dallo spirituale (KdZ 22). Sotto questo aspetto la sua posizione sembra preludere a quella degli scienziati "riduzionisti" del nostro tempo. Tuttavia l'enfasi che egli pone sulla nuova coscienza naturalistica e umanistica non è del tutto contenibile entro questo quadro. L'annuncio insistito di un "regno dell'uomo" e di una "salvezza", che comporta un appagamento totale del bisogno di felicità personale e collettivo, configura un'utopia dai tratti religiosi e tutt'altro che disincantati.

Le consolazioni e le promesse religiose sono diventate sempre più implausibili "per il popolo", che le vede in contraddizione con la vita reale. Ingannato dalla religione, che lo isola 
anche dagli altri uomini, questo soggetto deluso pare non abbia altre alternative che il "suicidio" o una disperata ricerca di salvezza mediante le proprie forze. Ora entrambe le opzioni vengono respinte da Friedrich. Contro ogni soluzione individualistica, egli proclama che occorre invece creare un nuovo legame. “C'è dunque un bisogno essenziale di fondaree diffondere una fede che unisca gli uomini con un vincolo (Band) più forte e prossimo di quello del cristianesimo, che promuova e sostenga cioè l'aspirazione, che ai nostri giorni si manifesta in molteplici modi, a integrare le insufficienze delle singole forze con uno spirito comune (Gemeischaftlichkeit)" (KdZ 41). Il vincolo dell'amore del prossimo - dice Friedrich riprendendo una tematica e un linguaggio originaria--- mente cristiano - si fonda sulla nostra "natura comune", e in particolare sul reciproco bisogno che proviamo l'uno dell'altro e che cispinge a considerarci "fratelli e sorelle nell'uomo". È il sangue che scorre in tutti i membri della famiglia umana a unirci, e non il sangue di Cristo che ci libera solo in qualità di coredenti (Miterlöste).

Per quanto riguarda le tipologie di "religioni senza dio" la posi-zione di Friedrich Feuerbach ci permette di avanzare l'ipotesi che anche la risoluzione del dio trascendente nella rete di relazioni (leggi) dell'universo, e della fede nell'etica, possa conservare una debole qualità religiosa; nel senso almeno che la stessa parola d'ordine della secolarizzazione sembra acquisire senso ed efficacia per chi vi si riconosce nella forma di una "fede".

\section{Bibliografia}

DAWKINS, R. The Good Delusion. London: Bantam Press, 2006.

DWORKIN, R. Religion without God. Cambridge: Harvard University Press, 2013.

FEUERBACH, F. Die Religion der Zukunft. Zürich: Verlag des Literarischen Comptoirs, 1845.

FEUERBACH, F. Die Religion der Zukunft: Die Bestimmung des Menschen. Nürnberg: Verlag von Theodor Cramer, 1845.

FEUERBACH, F. Die Religion der Zukunft: Mensch oder Christ?. Nürnberg: Verlag von Theodor Cramer, 1845.

FEUERBACH, F. Die Kirche der Zukunft. Bern, 1847.

FEUERBACH, L. Opere. Bari: Laterza, 1965. 
FEUERBACH, L. Ueber das 'Wesen des Christentums' auf Stirners 'Der Einzige und sein Eigentum'. Gesammelte Werke, a cura di W. Schuffenhauer. Berlin: Akademie Verlag, 1970.

FLORES D’ARCAIS, P.; ONFRAY, M.; VATTIMO, G. Atei o credenti?. Roma: Fazi, 2007.

FRAIJO, M. ¿Religión sin Dios? XXI Conferencias Aranguren. Isegoria, 47, 2012/2, p. 381-419.

ONFRAY, M. Traité d'athéologie. Paris: Grasset, 2006.

RADBRUCH, G. Die Feuerbachs. Eine Dynastie, in Gestalten und Gedanken. Acht Studien. Leipzig: Koehler \& Amelang, 1942.

SCRUTON, R. Il volto di Dio. Milano: Vita e Pensiero, 2013.

UNAMUNO, M. Del sentimento tragico della vita. Milano: Studio Editoriale, 2017.

Recebido em: 19/12/2020

Aprovado em: 27/3/2021

\section{Ferruccio Andolfi}

Professor emérito de Filosofia da História da Università degli Studi di Parma, Itália. Atuou como professor convidado nas universidades de Durham, Berkeley, Seatle, Freiburg e Berlin. Atualmente se ocupa com as relações entre humanismo e individualismo, com particular interesse pela história do pensamento do século XIX. É diretor da revista La società degli individui. 